\title{
Las aplicaciones de la teoría de juegos en la gerencia estratégica
}

\section{The applications of game theory in strategic management}

Ing. Jorge Brito, MBA.

Universidad Internacional del Ecuador, Ecuador

Autor para correspondencia: jurgenbritto@gmail.com

Fecha de recepción: 7 de Abril de 2016 - Fecha de aceptación: 25 de Mayo de 2016

\section{Resumen}

El presente trabajo tiene como objetivo conocer cómo la teoría de juegos puede ayudar a los directivos en el proceso de diseño e implementación de la estrategia de las organizaciones, que les permitan en corto y largo plazo general valor a los grupos de interés.

Palabras claves: teoría de juego; negocios; gerencia

\begin{abstract}
This paper aims to determine how game theory can help managers in the process of designing and implementing the strategy of organizations, enabling them generally short and long term value to stakeholders.
\end{abstract}

Keywords: game theory; business; management 


\section{Introducción}

Hasta finales del siglo XX, el éxito de la gestión directiva, estaba supeditado al desempeño de las organizaciones. Preparar a las organizaciones para responder con flexibilidad a los cambios del mercado del entorno, la incorporación permanente de mejores prácticas, análisis competitivos frecuentes versus la competencia, y el desarrollo de ventajas competitivas frente a la competencia, estaban dentro de los objetivos fundamentales de los gerentes. Sin embargo, en muchos sectores, no es precisamente así. Es necesario entonces, distinguir la eficiencia operacional y la estrategia (Porter, 1999).

La eficiencia operacional desarrolla el aprendizaje de las organizaciones, el conocimiento es considerado ahora como un factor de producción y pertenece a la teoría de la firma (Liebeskind, 1996) y su tratamiento debe ser estratégico (D’Alessio, 2010). La estrategia competitiva consiste en ser diferente; las organizaciones deben seleccionar de manera estratégica un conjunto de actividades distintas para entregar una mezcla única de valor (Porter, 1999).

Las organizaciones pueden evaluar posibles estrategias basadas en el conocimiento en función de las competencias diferentes. El desarrollo de las estrategias está ligadas al conocimiento y experiencia de los empleados y a la capacidad de trabajar en equipo. El desarrollo de la estrategia está supeditada a aspectos, tales como: circunstancias del entorno, situaciones internas de la organización, posiciones políticas, entre otras. Adicionalmente y quizás el punto central del desarrollo estratégico y su implementación, depende del estilo de liderazgo del líder (Bill, 1994).

\section{La gestión estratégica y los tipos de liderazgo}

La literatura de la gestión estratégica, sugiere que la toma de decisiones de los líderes esta supeditada a su experiencia previa, a su formación profesional y capacitación, la perspectiva personal sobre lo que significa el trabajo, entre otras. Bill (1994) propone la siguiente clasificación para los diferentes tipos de líderes: 1) el administrador clásico, el planificador, 3) el gerente, 4) el líder de respuestas políticas, 5) el competitivo, 6) facilitador y auto organizado, 7) estratega de cambios, y 8) sensible a las crisis.

Las decisiones estratégicas de las organizaciones están relacionadas también con los estilos de liderazgo y de acuerdo a Bill (1994) tienen los siguientes dominios: 1) la existencia de los procesos: procesos formales, tales como los relacionados a políticas definidas y aceptadas por la dirección en cuanto al manejo de los clientes, líneas de crédito, contratación de colaboradores, administración de proveedores; e informales, tales como participación de los colaboradores en proyectos por afinidad y experiencia, líneas de reportes, entre otras; 2) el ambiente de trabajo y la motivación de los colaboradores y las relaciones interpersonales; 3 ) los problemas identificados y propios del negocio; 4) el estado actual de los procesos, las relaciones de poder; 5) la perspectiva del líder y la perspectiva individual y del grupo gerencial, 6) patrón de relacionamiento y 7) objetivos de la dirección.

A pesar de la creciente necesidad de un enfoque más amplio que considere los estilos del líder para aplicar sus conocimientos a la gestión estratégica, las investigaciones demuestran que los problemas, su abordaje y las decisiones de los gerentes están relacionadas a sus propio estilo de liderazgo, con frecuencia, se descubre que gerentes y directivos asumen una postura 
manifiesta o implícita que la decisión tomada es "la mejor decisión por el momento". La tendencia en la literatura de gestión estratégica es presentar una variedad de contextos estratégicos con una serie de situaciones particulares propias de cada negocio. El análisis conduce a concluir que los líderes toman sus decisiones de acuerdo a su propio estilo (Bill, 1994).

Según Rumelt, Schendel y Teece (1991), la estrategia competitiva seguirá siendo parte integral de la gestión estratégica y que su relación con la economía va a evolucionar y asumir nuevas formas en el futuro. Graetz (2002), propone que la planificación estratégica y el pensamiento crítico son diferentes, pero están implícitamente relacionadas. Según la autora, el rol de la planificación estratégica es ejecutar las tácticas desarrolladas a partir del proceso de pensamiento crítico y lograr su integración en el negocio. La planificación estratégica tiene las siguientes características: es lógica, sistemática, convencional, prescriptiva y convergente. El rol del pensamiento crítico dentro de la estrategia es buscar innovar, romper esquemas tradicionales y generar aportes diferentes para liderar la industria en que participa la empresa. El pensamiento crítico sugiere: creatividad, intuición, innovación, divergencia.

Los aspectos más importantes de la investigación de Graetz (2002) en el contexto de la relación entre el las habilidades y la inteligencia emocional de los líderes organizacionales, es la capacidad de promover el pensamiento crítico en las empresas.

Goleman (1998) encontró que los resultados de las organizaciones están altamente relacionados con la inteligencia emocional del líder. Las principales características de la inteligencia emocional y liderazgo son: 1) altas relaciones interpersonales, 2) apertura al cambio, 3) habilidad para transmitir la nueva visión del negocio, 4) compromiso y entusiasmo, 5) creer en las habilidades de los seguidores, 6) desarrollo de redes de relacionamiento, 7) motivación permanente, y 8) entendimiento de la operación del negocio.

La investigación académica y empresarial, se enfoca en conocer qué elementos están relacionados en el mejoramiento del desempeño de las organizaciones. Una perspectiva diferente, se vincula con la teoría de juegos, se presenta los criterios de algunos investigadores quienes proponen que el entendimiento de esta teoría podría generar ventajas a los líderes de las empresas para tomar decisiones en contextos de alta competitividad.

\section{La teoría de juegos}

El éxito de la teoría de juegos en otras disciplinas como la economía específicamente, ha sugerido a los investigadores el análisis de ésta en las ciencias sociales. A continuación, se presentan algunos conceptos fundamentales para comprender la misma. Para Camerer (1991) un juego se constituye con varios jugadores que eligen sus estrategias de juego en forma individual. Sus decisiones estratégicas implican consecuencias, que generan pagos compensaciones, es decir, cuando un jugador toma su decisión, ésta, le significará: ganar o perder algo. Un juego repetido está compuesto por varias etapas que implican decisiones diferentes. El equilibrio es el resultado del juego o un conjunto de resultados.

El autor manifiesta que es importante distinguir los tres elementos que forman parte de esta teoría: los juegos, el razonamiento desarrollado para realizar la jugada o decisión y los 
puntos de equilibrio. Un aspecto que preocupa, incluso a los expertos en la teoría, es la confusión generalizada sobre la racionalidad requerida para que los jugadores encuentren el equilibrio. A menudo el conocimiento común de los jugadores es más que suficiente para justificar el equilibrio. Los jugadores pueden tomar decisiones de equilibrio, basados en su racionalidad respecto del juego o considerar que los otros jugadores harán lo propio.

La forma de jugar o de tomar las decisiones de los jugadores, también están supeditadas al estilo de liderazgo de cada uno. Por ejemplo: dos empresas que compiten en el mismo mercado -las telefónicas en caso de Ecuador- cada una tiene que tomar decisiones respecto al precio de venta de un producto que lanzará al mercado; el análisis de precio tendría que considerar cómo la competencia reaccionará una vez que salga al mercado (la primera jugada), desde el punto de vista racional, ambas empresas deberían tener el mismo afán: maximizar las utilidades. Sin embargo, que podrían pensar ambas empresas sobre la participación de mercado, la decisión de la segunda empresa, ¿tendrá el mismo objetivo? Esta idea de que un individuo racional, es aquel que maximiza la utilidad de la empresa cuando posee información suficiente para genera equilibrios sensibles, es analizada por la teoría de juegos moderna, ésta, tiene interés por la naturaleza de la conducta racional.

Para Rumelt, et. al (1991), la teoría de juegos aplicada a la organización industrial tiene interés en dos temas básicos: el compromiso con las estrategias y la reputación. El compromiso, está relacionado con aspectos tales como: la inversión en activos, el exceso de capacidad instalada, la investigación, las mermas, y la estructura financiera. La reputación del líder empresarial, está relacionada a sus decisiones previas en los juegos, lo importante de este aspecto son las creencias que puedan tener la contraparte.

Camerer (1991) sugiere que la teoría de juegos es una muy buena opción para analizar y resolver las implicaciones lógicas de directivos que piensan seriamente las consecuencias de sus decisiones. Sin embargo, identifica cuatro aspectos que les preocupa a los investigadores de estrategia: 1) la dificultad para el uso de los modelos de teoría de juegos, 2) la poca generalización de los supuestos, 3) la poca confiabilidad de las pruebas dadas las características transversales de la información, y 4) los aspectos sorpresa o no esperados por parte de los tomadores de decisión.

La teoría de juegos, genera la atención de los expertos y estrategas, es un tema de acción y reacción, la innovación es intrínsecamente dinámica y los procesos también, los líderes tienen permanentes desafíos y uno de los más representativos son los resultados de su gestión estratégica. Un campo de oportunidad para la inclusión de la teoría de juegos en las organizaciones es la relación producto-mercado y el desarrollo de las capacidades de la organización (Rumelt, et. al., 1991).

Respecto a la validez de los modelos de la teoría de juegos, Saloner (1991) argumenta que existe un gran potencial en el desarrollo de modelos metafóricos que podrían capturar las amplias características cualitativas de las interacciones estratégicas. Para el efecto presentó un estudio que analiza los atributos positivos de la modelización matemática y el papel de los modelos de teoría de juegos y su potencial para apoyar estudios empíricos al respecto. 
La literatura contempla importantes avances de la teoría de juegos como base para la toma de decisiones directivas. Cada vez más, y a partir de complejos modelos matemáticos, que incorporan variables cualitativas sobre las preocupaciones de los líderes respecto a las decisiones. El desarrollo de los modelos supone que a los líderes empresariales del mismo sector, les preocupan o están evaluados por índices de gestión estratégica similares, por lo tanto bajo circunstancias específicas y el conocimiento racional de los intereses de las organizaciones y de los propios del líder, se podría inferir una decisión en cierto sentido.

Por otro lado, en función del conocimiento de los patrones recabados a través de estudios longitudinales de líderes competitivos y específicamente de los procesos de decisión y experiencia, podría predecirse una decisión. Evidentemente, no existen garantías sobre este tipo de análisis y decisiones, sin embargo los avances de la teoría de juegos presentados en los modelos propuestos por Saloner (1991) suponen que un avance importante en la relación de la teoría y la gestión estratégica. Se requerirá de más investigaciones empíricas para validar los modelos, los mismos que soportados por programas computacionales, éstos podrían predecir la forma de jugar o las decisiones de los líderes en algunas sectores de la economía. Un aspecto importante, no considerado en la teoría de juegos que podría limitar su validez en áreas de interés social, está relacionada al comportamiento de los seres humanos, ya que existe evidencia empírica que no hay garantía sobre la coherencia de sus decisiones.

Bibliografía

Bill, R. (1994). Comprehensive Approach to Strategic Management: Leading across the Strategic Management Domain. Management Decision, 32(8), 27-41.

Camerer, C. F. (1991). Does Strategy Research need Game Theory? Strategic Management Journal, $12,137-152$.

D’Alessio, F. (2010). Liderazgo y atributos gerenciales: una visión global y estratégica. México, Pearson Educación Prentice Hall.

Goleman, D. (1998). What make a leader?, Harvard Business Review, November/December, 93-102.

Graetz, F. (2002). Strategic Thinking versus Thinking Planning: Towards understanding the complementarities. Management Decision, 40 (5/6), 456-462.

Liebeskind, J. P. (1996). Knowledge, strategy, and the theory of the firm. Strategic Management Journal, 17(Winter Special Issue), 93-107.

Porter, M. E. (1999). ¿Qué es la estrategia?, Harvard Business Review, 100-117.

Rumelt, R. P., Schendel, D., \& Teece, D. J. (1991). Strategic Management and Economics. Strategic Management Journal, 12, 5-29.

Saloner, G. (1991). Modeling, Game Theory, and Strategic Management. Strategic Management Journal, 12, 119-136. 